Kunst und Kultur sind Merkmale und Ausdruck des Menschseins. Recht als Grundordnung menschlichen Zusammenlebens ist vielfach mit innen befasst. Kultur- und kunstrechtliche Fragestellungen finden sich im Privat-, Straf- und Öffentlichen Recht, im Internationalen Privatrecht, im Völker- wie Europarecht.

Die „Schriften zum Kunst- und Kulturrecht “ verstehen sich als Forum für kulturell-juristische Themen im deutschsprachigen Raum. Sie vereinen Werke aus allen Rechtsgebieten der drei Rechtsordnungen Deutschlands, Österreichs und der Schweiz sowie aus dem Völkerund Europarecht und dokumentieren auf diese Weise die Bedeutung und Vielgestaltigkeit des Kunst- und Kulturrechts.

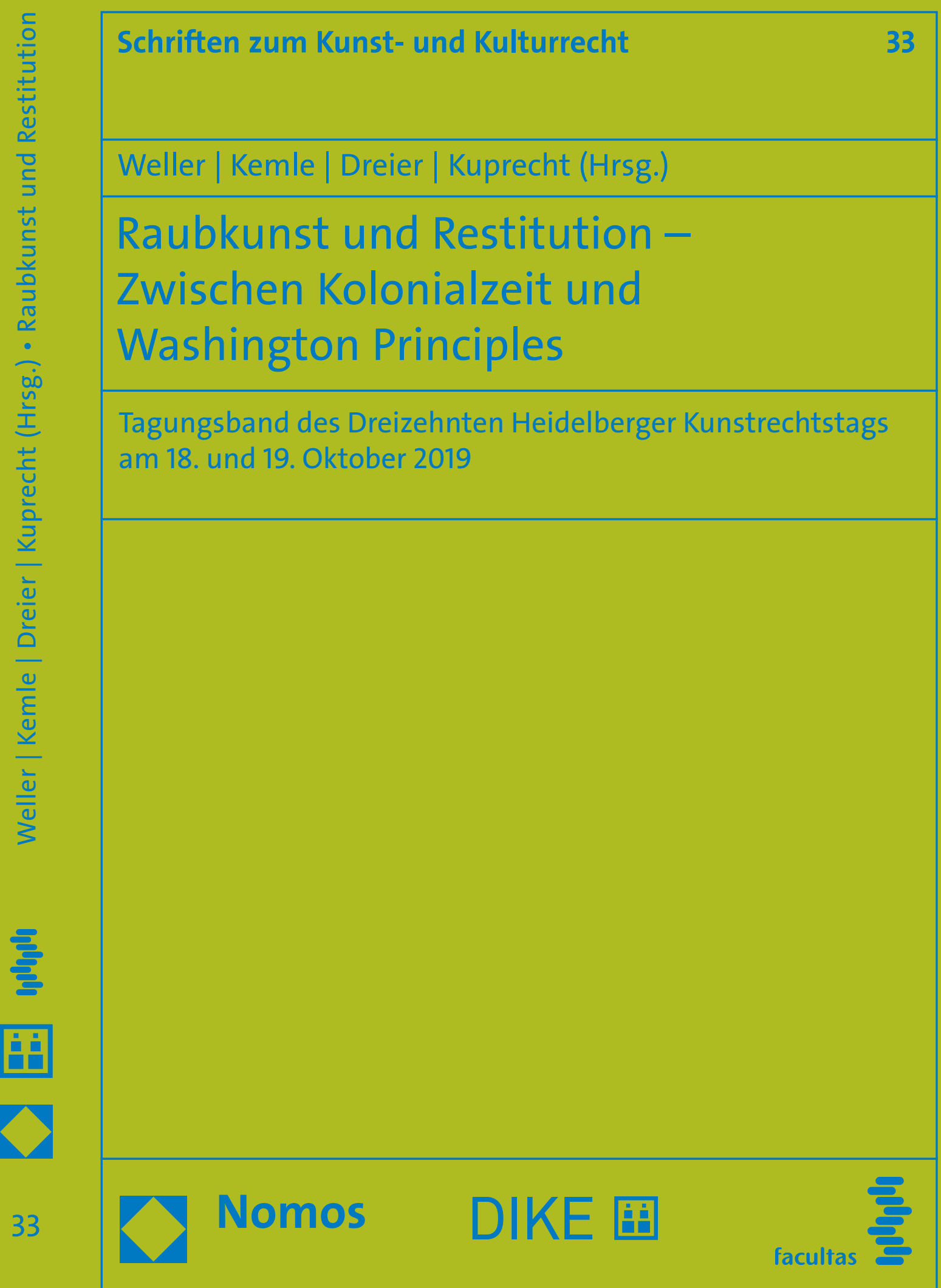




\section{Schriften zum Kunst- und Kulturrecht}

Herausgegeben von

Prof. Dr. Kerstin von der Decken, Universität Kiel

Prof. Dr. Frank Fechner, Technische Universität IImenau

Prof. Dr. Dres. h.c. Burkhard Hess,

Max Planck Institute Luxembourg for International,

European and Regulatory Procedural Law

RA Prof. Dr. iur. Dr. phil. h.c. Peter Michael Lynen,

NRW Akademie der Wissenschaften und der Künste

Prof. Dr. Rainer J. Schweizer, Universität St. Gallen

Prof. Dr. Armin Stolz, Universität Graz

Prof. Dr. Matthias Weller, Mag. rer. publ., Universität Bonn

Band 33

unterstützt durch

Institut für Kunst und Recht IFKUR e.V.

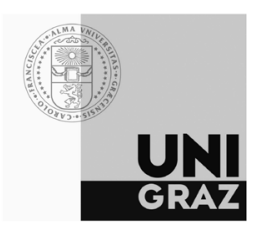




\section{Matthias Weller | Nicolai B. Kemle | Thomas Dreier Karolina Kuprecht (Hrsg.) \\ Raubkunst und Restitution - Zwischen Kolonialzeit und Washington Principles}

Tagungsband des Dreizehnten Heidelberger Kunstrechtstags am 18. und 19. Oktober 2019

\section{Nomos


nlineversion

Nomos elibrary

Die Deutsche Nationalbibliothek verzeichnet diese Publikation in der Deutschen Nationalbibliografie; detaillierte bibliografische Daten sind im Internet über http://dnb.d-nb.de abrufbar.

ISBN 978-3-8487-7034-2 (Print)

ISBN 978-3-7489-1157-9 (ePDF)

ISBN 978-3-03891-282-8 (Dike Verlag, Zürich/St. Gallen)

ISBN 978-3-7089-2067-2 (facultas Verlag, Wien)

1. Auflage 2020

(c) Nomos Verlagsgesellschaft, Baden-Baden 2020. Gesamtverantwortung für Druck und Herstellung bei der Nomos Verlagsgesellschaft mbH \& Co. KG. Alle Rechte, auch die des Nachdrucks von Auszügen, der fotomechanischen Wiedergabe und der Übersetzung, vorbehalten. Gedruckt auf alterungsbeständigem Papier. 
Inhalt

Der Kunstsammler und das Kunstrecht

Erik Jayme

Provenienz - Geschichte und Perspektiven eines neuen Paradigmas in den Geistes- und Kulturwissenschaften

Christoph Zuschlag

Eine Frage des Wertes. Kunstwerke im Fadenkreuz der Judenverfolgung im Nationalsozialismus

Ulrike Saß

Warum ein „Restatement of Restitution Rules for Nazi-Confiscated Art“? Das Beispiel „Fluchtgut“

Matthias Weller / Anne Dewey

Zwischen Post- und Neokolonialismus - Geistiges Eigentum für traditionelles Wissen, traditionelle kulturelle Ausdrucksformen und indigene Ressourcen

Thomas Dreier

Geistiges Eigentum und „traditionelle“ Kunst

Andreas Rabmatian

Kulturgüter aus der Kolonialzeit und Restitution: Änderungen ohne Änderungen

Karolina Kuprecht

The Bangwa Queen: Artifact or Heritage?

Evelien Campfens 


\title{
Geistiges Eigentum und „traditionelle“ Kunst
}

\author{
Andreas Rahmatian"
}

\section{Einleitung: Worum geht es beim Schutz „traditioneller" Kunst?}

Seit etwa der Jahrtausendwende ist die Kommerzialisierung besonders nicht-europäischer indigener Kulturen vielen ein Dorn im Auge; ein ökologischeres und stärkeres anti-koloniales Denken mögen dazu beigetragen haben. Das ist ja auch grundsätzlich zu begrüßen. Warum sollen multinationale Unternehmen traditionelle Textilmuster indigener Völker für ihre massenproduzierte Kleidung verwenden können, obwohl die Nutzung solcher Muster aus ihrem kulturellen, sozialen und religiösen Zusammenhang gerissen wird und die indigenen Völker keinen oder einen nur unbedeutenden Anteil am Profit aus dem Verkauf erhalten? ${ }^{1}$ Bei moderner Kunst wird das Problem allerdings schwieriger. Kann ein afrikanischer Künstler die graphischen Formen und Farben der traditionellen Hausfassadenmalereien der Ndebele im südlichen Simbabwe und in Südafrika in seiner Skulptur verwenden?² Haben die Ndebele ausschließliche Rechte daran, und wenn ja, wer genau innerhalb der Ndebele? Können Lizenzen vergeben werden (und an wen?), oder ist die Benützung frei? Muss ein Künstler für die Benützung den Ndebele angehören? Oder kann etwa eine Künstlerin, die den Shona (Mehrheitsbevölkerung in Simbabwe) angehört, diese „traditionelle“ Kunst ebenfalls verwenden? Ein Südafrikaner auch? Auch ein Weißer oder muss der Künstler schwarz sein? Oder auch eine Ni-

* Prof. Dr. Andreas Rabmatian, University of Glasgow, School of Law, UK.

1 Generell Helfer/Austin 2011, 432-433. Maasai v. Louis Vuitton, siehe https://-www.i ndependent.co.uk/life-style/fashion/maasai-people-cultural-appropriation-luxury-fa shion-retailers-louis-vuitton-east-africa-intellectual-a7553701.html (aufgerufen am 2. Februar 2020). Siehe einen thematisch ähnlichen Fall, Bildrechte und Persönlichkeitsrechte betreffend, vor dem High Court von Uganda, Asege Winnie v. Opportunity Bank (U) Ltd \& Anor [2016] UGCOMMC 39, und https://ipkenya.word press.com/2016/04/29/commercial-appropriation-of-perso-nality-and-image-rights-p rotection-uganda-high-court-decision-in-asege-winnie-v-opportunity-bank/\#more-6 648 (aufgerufen am 4. Februar 2020).

2 Das selbständige Matabele-Königreich unter dem letzten König Lobengula wurde von den Briten zwischen 1893-96 zerschlagen, siehe Hallett 1974, 495-499. 
gerianerin (immerhin eine Afrikanerin) oder sogar Europäer und Nordamerikaner?

Diese Fragen sind einem vielleicht unangenehm und das sollten sie auch sein. Sie umgrenzen das Thema dieses Beitrages. Denn „Tradition“ und "traditionelle Kunst" sind immer normative Begriffe, jedenfalls wenn sie Schutzrechte genießen sollen, und damit stellt sich sofort die Frage, wer diese - vielleicht urheberrechtsähnlichen - Schutzrechte beanspruchen kann und wie man potenziell Berechtigte feststellt. Das eröffnet die Möglichkeit einer juristisch abgesicherten identitären und rassistischen Segregation, wie sie aus der Geschichte hinreichend bekannt ist. Diese Probleme, mit Beispielen besonders aus dem afrikanischen Kontinent, werden im Folgenden diskutiert werden.

\section{Ein Beispiel aus Kenia}

Internationale und nationale Regelungen für den Schutz indigener Kunst, oder Ausdrucksformen traditioneller Kulturen, existieren und werden laufend erweitert. Wie effizient sie sind, ist fraglich, aber im Lichte der hier präsentierten Argumentation ist die Wirksamkeit solcher Regelungen gar nicht wünschenswert. Ein sehr neues Beispiel für eine Einführung eines Schutzes „indigener" Werke ist Kenia.

Im September 2019 wurde in Kenia eine Novelle (Copyright (Amendment) Act No. 20 of 2019) zum Urheberrechtsgesetz 2001 (Copyright Act of 2001) erlassen, ${ }^{3}$ das, neben anderen Änderungen, eine sui-generis Schutzregelung für "traditional knowledge“ und "cultural expressions“ einführt (oder eigentlich verstärkt) und den im Urheberrechtsgesetz bis dahin verwendeten Begriff „Folklore“ eliminiert. Dies ist eine Anpassung an Kenias bereits existierenden „Protection of Traditional Knowledge and Cultural Expressions Act 2016“, der „cultural expressions“ in $\$ 2$ sehr detailliert definiert: ${ }^{4}$

„,cultural expressions' means any forms, whether tangible or intangible, in which traditional culture and knowledge are expressed, appear or are manifested, and comprise of the following forms of expressions or combinations thereof."

3 Das Gesetz trat am 2. Oktober 2019 in Kraft.

4 Siehe: http://kenyalaw.org/kl/fileadmin/pdfdownloads/Acts/ProtectionofTraditio-n alKnowledgeandCulturalExpressionsAct_No33of2016.pdf (aufgerufen am 26. Januar 2020). 
Diese sind demnach: (a) verbal expressions ... (b) musical expressions ... (c) movement and dance ... (d) visual art in the widest sense, including woodwork, textiles, needlework, musical instruments, maps and architectural forms.

„Traditional knowledge“ wird in demselben $₫ 2$ definiert als:

„any knowledge (a) originating from an individual, local or traditional community that is the result of intellectual activity and insight in a traditional context, ... or (b) contained in the codified knowledge systems passed on from one generation to another ..."

Die Rechte liegen bei den „owners and holders of traditional knowledge“ $(\mathbb{9} 9)$, beziehungsweise bei den „owners und holders of cultural expressions“ $(\$ 16)$, und diese sind die „community“ $(\$ \$ 10,14)$, welche folgendermaßen definiert wird $(\$ 2)$ : „,community“ means a homogeneous and consciously distinct group of the people who share any of the following attributes: (a) common ancestry; (b) similar culture or unique mode of livelihood or language; (c) geographical space; (d) ecological space; or (e) community of interest."

Die Novelle (Copyright (Amendment) Act 2019) zu Kenias Urheberrechtsgesetz greift dieses Gesetz von 2016 insoweit auf, als es in $\$ 2(e)$ den urheberrechtlichen Begriff der „Folklore“ des \2(1) des Gesetzes von 2001 abschafft und damit indirekt auf die spezielle Regelung des Gesetzes von 2016 verweist, sodass der Schutz von Werken traditioneller Kultur nicht mehr dem Urheberrecht, sondern ausschließlich dem Spezialrecht von 2016 unterliegt.

Das Gesetz Kenias von 2016 ist eine moderne, sehr sorgfältige und detailreiche Umsetzung internationaler Bestrebungen, „Traditional Knowledge“ und „Traditional Cultural Expressions" zu definieren und zu schützen. Es unterscheidet zwischen „Traditional Knowledge“ und „Traditional Cultural Expressions“, und definiert die potenziellen Nutznießer eines Schutzes dieses indigenen kulturellen Ausdrucks als „community“. Gleichzeitig lässt es die grundsätzlichen Probleme einer solchen Schutzphilosophie erkennen, die man auch auf internationaler Ebene findet. Die generell verwendeten internationalen Definitionen für „Traditional Cultural Expressions" zeigen die Schwierigkeiten deutlich.

III. Versuch einer Arbeitsdefinition und Skizzierung internationaler Regelwerke

Im Zusammenhang mit (traditioneller oder moderner) Kunst interessieren uns hier „Traditional Cultural Expressions“ (TCEs). „Traditional Knowl- 
edge“ (TK) ist ein weiterreichender Begriff, der von der World Intellectual Property Organisation (WIPO) mit „knowledge, know-how, skills and practices that are developed, sustained and passed on from generation to generation within a community, often forming part of its cultural or spiritual identity" umschrieben wird; 5 eine international allgemein anerkannte Definition gibt es allerdings nicht. ${ }^{6}$ TCEs sind eher auf Werke der Literatur, Kunst und Wissenschaft (in einem weiteren Sinn verstanden), also Sachgebiete, die westliche Juristen eher im Bereich des Urheberrechts ansiedeln. ${ }^{7}$ Oft werden TK und TCE unter dem Oberbegriff Indigenous Cultural Heritage $(\mathrm{ICH})$ zusammengefasst, oder TCE wird als eine Untergruppe von TK gesehen: eine genaue Einteilung ist schon deshalb nicht zielführend, weil das Objekt der Einteilung, das "traditionelle Wissen“, sich inhaltlich westlichen Definitionskriterien oft entzieht.

Das vielleicht wichtigste internationale Instrument für den Schutz von TCEs, die UNESCO-Konvention über den Schutz und die Förderung der Vielfalt kultureller Ausdrucksformen (Convention on the Protection and Promotion of the Diversity of Cultural Expressions, 2005, in Kraft seit 2007) $)^{8}$ ordnet einen sui generis-Rechtsschutz von TCEs an: individuelle subjektive Rechte erfließen aus dieser Konvention nicht; es sind vielmehr die Staaten angehalten, entsprechende Regelungen zu erlassen. Der ältere Begriff „Folklore" wird international nicht mehr, aber in nationalen Gesetzen (wie bis vor kurzem etwa in Kenia) noch weiter verwendet. Es existieren weitere für den TCE-Schutz relevante internationale Konventionen, ${ }^{9}$ die aber für die hier interessierende Diskussion nichts Neues enthalten.

Zusammenfassend kann man TCEs und ihren Rechtsschutzmechanismus also mit der folgenden Arbeitsdefinition (in Anlehnung an eine Defi-

5 Siehe WIPO Website: https://www.wipo.int/tk/en/tk/ (aufgerufen am 26. Januar 2020).

6 Vgl. Dutfield 2017, 145. Generell über das Problem der Definition in diesem Bereich siehe etwa Drahos/Frankel 2012, 9.

7 Rabmatian 2019, 162. In Bezug auf die Pazifischen Inseln, wo diese hier angewendete Trennung zwischen TK und TCEs stimmt, siehe Forsyth 2012, 190. Die Einteilung TK/TCEs ist nicht immer zwingend.

8 Siehe UNESCO Website: http://portal.unesco.org/en/ev.php-URL_ID=31038\&-UR L_DO=DO_TOPIC\&URL_SECTION=201.html (aufgerufen am 26. Januar 2020). Es gibt keinen authentischen deutschen Text (Konventionssprachen sind Englisch, Französisch, Spanisch, Arabisch, Chinesisch und Russisch), daher wird die englische Fassung benützt.

9 Siehe Petrillo 2019, 241-248. Ein Überblick auch bei zB bei Rabmatian 2019, 164-165 mwN. Über das UN Human Rights Committee und indigene Rechte, siehe Helfer/Austin 2012, 438-441. 
nition von WIPO $)^{10}$ umreißen: TCEs bezeichnen Wissenssysteme, Kreationen, Innovationen und kulturelle Ausdrucksformen, die im Allgemeinen von Generation zu Generation weitergegeben werden und sich normalerweise auf ein bestimmtes Volk oder sein Gebiet beziehen. Relevant sind hier - neben landwirtschaftlichem, technischem, ökologischem und medizinischem Wissen - Musik, Tanz, Liedkunst, Ornamente und (Textil-)Muster, Geschichten und Kunstwerke, Elemente von Sprachen wie Namen, geografische Angaben und Symbole, sowie bewegliche Kulturgüter. Diese einmal festgestellten TCEs können dann einem (teilweisen) Urheberrechtsschutz unterliegen, oder - das ist die modernere Version - einen sui generisSchutz genießen, der allerdings seiner Natur nach auch dem ,geistigen Eigentum", also vor allem dem Immaterialgüterrecht des Urheberrechts, nachgebildet ist. ${ }^{11}$ Die Rechteinhaber sind eine einigermaßen deutlich umgrenzte "traditional community“, die sich durch gemeinsame Abstammung, das Teilen eines gemeinsamen geographischen Lebensraumes und eine spezifische Art der Lebensgestaltung, und durch eine ähnliche Kultur oder Sprache auszeichnen soll. ${ }^{12}$ Wer aber genau innerhalb dieser „traditionellen Gemeinschaft" einen Rechtsanspruch erhalten soll und wie dieser ausgestaltet ist, bleibt oft im Dunkel, ${ }^{13}$ oder es werden staatliche Rechtevertreter gesetzlich angeordnet, die als Nutznießer, anscheinend stellvertretend für die indigenen Völker, operieren sollen - etwa der Staatspräsident $^{14}$ - oder die TCEs sind überhaupt im Staatseigentum. ${ }^{15}$

Das obige Beispiel aus der Gesetzgebung Kenias ist eine gute Illustration eines solchen Schutzsystems auf nationaler Ebene.

10 WIPO Report on Fact-finding Missions on Intellectual Property and Traditional Knowledge (1998-1999): Intellectual Property Needs and Expectations of Traditional Knowledge, Seite 25, https:/www.wipo.int/edocs/pubdocs/en/tk/768/wi-po_p ub_768.pdf (aufgerufen am 26. Januar 2020).

11 Weitere Diskussion in Rabmatian 2019, 162-163. Bezüglich der Frage der - in der wissenschaftlichen Literatur oft reichlich übertriebenen - Figur des („romantischen“) Autors als den notwendigen Schöpfer für das (auch traditionelle?) Werk und als (ersten) Rechteinhaber für das System des Urheberrechtsschutzes, siehe Rabmatian 2007, 210-220.

12 Girsberger/Müller 2012, 179-180.

13 Siehe zB Burkina Faso als ein Beispiel für eine institutionelle Struktur des Kulturgüter- und TCE-Schutzes bei Traore 2019, 120.

14 So in Ghana, $\mathbb{S}$ 4(2), 17 Ghana Copyright Act 2005.

15 So etwa in Tansania, $\$ \$ \$ 25,29$ Tanzania Copyright and Neighbouring Rights Act 1999. 


\section{Rechtsschutz als juristisches Hindernis für die Entfaltung moderner Kunst}

Das erste offensichtliche Problem eines solchen, anfänglich sicher gut gemeinten, Rechtsschutzmechanismus ist die Idee einer „traditionellen“ Kultur und Kunst und ihren Ergebnissen, die das Objekt eines solchen Rechtsschutzes sein sollen. Die Debatte über die Bedeutung des Begriffes „traditionell“ und „traditionelle Kunst" sollte auf Künstler und Kunstkritiker, und in weiterer Folge Kunstwissenschaftler und Anthropologen, ob indigen oder westlich, beschränkt bleiben. In dem Moment, in dem eine Rechtsordnung Schutzregelungen für traditionelle und indigene Kultur gewährt, wird der Begriff der ,indigenen Tradition“ normativ. Man wird vielleicht erstaunt sein, dass zuvor bloß auf diverse Arbeitsdefinitionen internationaler Organisationen und Konventionen verwiesen wurde, aber kaum der Versuch unternommen wurde, den Begriff der „Tradition“ genauer zu definieren. Das ist auch nicht erforderlich, denn ein normativer Traditionsbegriff ist unabhängig von einer tatsächlichen, vielleicht durch Konsens in einer öffentlichen Diskussion festgestellten, Definition einer Tradition (falls dies möglich sein sollte). Gesetzesdefinitionen, authentische Interpretationen und juristische Fiktionen mögen (und rechtspolitisch gesehen: sollten) eine Ähnlichkeit mit der Realität aufweisen, aber das ist juristisch nicht notwendig: wenn eine Rechtsnorm anordnet, dass im Sinne eines bestimmten Gesetzes ein Hund als eine Katze zu verstehen ist, so werden Hunde rechtlich insofern wie Katzen behandelt. Was rechtlich eine (schutzwürdige) Tradition ist, wird durch Gerichtsentscheidungen autoritativ festgelegt werden müssen, vielleicht nach einem vorhergehenden Sachverständigenbeweisverfahren, also ähnlich einem Verfahren, in dem ausländisches oder Gewohnheitsrecht geltend gemacht wird, bei denen der Satz iura novit curia ${ }^{16}$ nicht gilt. ${ }^{17}$ All dies unterstellt, dass das Rechts- und Gerichtswesen funktionsfähig und sowohl in Hinblick auf die Sachverständigen als auch die Entscheidungsorgane frei von Korruption ist

16 Dies betrifft jedenfalls das inquisitorische Verfahren kontinentaleuropäischer Zivilprozesse, im adversalen Prozess des Common Law gilt diese Regel keineswegs generell.

17 Man wird vielleicht einwenden, dass ausländisches Recht und Gewohnheitsrecht gegenüber dem nationalen Recht als zu beweisende Tatsachen behandelt werden, aber dem Wesen nach normativ sind, während eine kulturelle Tradition eine "echte" feststellbare Tatsache ist. Das stimmt nur zum Teil, weil in den eher „künstlerischen“ Teil (zB Musik, Tanz, Epik und Poesie der Oraltraditionen) indigener Traditionen oft auch stark gewohnheitsrechtliche Elemente eingeflochten sind. Siehe zB Forsyth 2012, 195-196 bezüglich der pazifischen Inseln. Zur Feststellung von (inländischem) afrikanischem Gewohnheitsrecht, siehe Allott 1960, 74. 
- eine geradezu abenteuerlich naive Annahme für viele Länder, die solche indigene Traditionen besitzen.

In jedem Prozess, der eine Verletzung der geschützten Tradition zum Gegenstand hat, entweder auf der Basis des Urheberrechts, oder - heute häufiger - auf der Grundlage eines sui generis-Schutzes, muss die Existenz der geschützten Tradition oder eines für diesen Prozess relevanten Aspektes als Grundlage für die Rechtsverletzung festgestellt werden. Das ist im Wesentlichen nicht anders als in einem Patenteingriffs- oder Urheberrechtsverletzungsverfahren, in dem der Beklagte die Ungültigkeit des $\mathrm{Pa}$ tents behauptet oder den Urheberrechtsschutz des fraglichen Werkes bestreitet. Der Unterschied ist freilich, dass eine „patentierte Erfindung“ oder ein "urheberrechtlich geschütztes Werk" im Vergleich recht genau umrissene Rechtsbegriffe sind, die sich auf entsprechende Tatsachen anwenden lassen, während „Tradition“ (oder irgendein Aspekt, zB ein indigenes Muster) oft sehr schwammig sein kann und einen ausgedehnten Schutz verlangt, der weit über den eines klassischen Eigentumsschutzes hinausgeht. Darüberhinaus ist es kaum möglich, eine solche rechtlich geschützte Tradition anders als ein willkürliches juristisches Konstrukt zu interpretieren, das nicht viel mit einer „gelebten“ Tradition zu tun hat und sogar völlig von ihr losgelöst sein kann. Diese normative Konstruktion kann aber zur politischen und wirtschaftlichen Machtausübung herangezogen werden. ${ }^{18}$

Es mag eingewendet werden, dass ein derartiger rechtsdogmatisch-prozessoraler Zugang, der sich außerdem vom klassischen Immaterialgüterrecht ableitet, für den Schutz indigener Traditionen und ihre TCEs unpassend ist, und es gibt tatsächlich sehr selten „konventionelle“ Zivilprozesse in Bezug auf indigene traditionelle Werke. ${ }^{19}$ Das liegt nicht nur daran, dass Betroffene ihre Rechte nicht kennen, oder nicht die finanziellen Mittel haben, sie durchzusetzen, sondern auch, dass viele Schutzsysteme für TCEs einen unklaren oder, im Falle internationaler Konventionen, überhaupt keinen Durchsetzungsmechanismus besitzen. Das macht die internationale und teilweise auch nationale Gesetzgebung für den Schutz von TCEs zu wirkungslosen Absichtserklärungen, und das ist, wie man überraschenderweise feststellen muss, auch gut so. Wäre der TCE-Schutz ähnlich wirksam wie der Schutz von gewöhnlichen Immaterialgüterrechten, so könnte dies katastrophale Auswirkungen haben.

18 Siehe unten unter $\mathrm{V}$.

19 ZB John Bulun Bulun and another v. R. \& T. Textiles Pty. Ltd. [1998] FCA 1082 (Federal Court of Australia). 
Die Schwierigkeiten beginnen damit, dass Traditionen von dem Paradox leben, dass sie ewiggültige Leitprinzipien darstellen wollen („unsere Vorväter haben dies schon seit Jahrhunderten so gemacht"), sich gleichzeitig aber über die Zeiträume erheblich verändern, auch wenn dies vielleicht nicht anerkannt wird oder werden soll. Werden Tradition oder TCEs, etwa in einem Verfahren, normativ festgestellt, dann sind sie rechtlich festgefroren und schließen wenigstens theoretisch eine Weiterentwicklung aus, unabhängig davon, ob diese Legaldefinitionen die Tradition überhaupt widerspiegeln und nicht ohnehin ein künstliches Konstrukt darstellen. Die Frage, ob Traditionen generell nicht überhaupt erfunden sein können, ${ }^{20}$ stellt sich hier nicht, denn sowie eine Tradition oder ihr Ausdruck normativ definiert sind, ist sie sowieso „erfunden“ und losgelöst von ihrem soziokulturellen Hintergrund. Natürlich entwickelt sich auch das Recht weiter, nur hinkt es sozio-kulturellen Entwicklungen typischerweise um Jahrzehnte hinterher. Ein anschauliches Beispiel ist das englische Common Law, das letztlich ein durch Präzedenzfälle festgestelltes, sodann festgelegtes Gewohnheitsrecht ist, auch wenn englische Juristen dies meiner Erfahrung nach nicht so explizit wahrnehmen. ${ }^{21}$ Ein als veraltet empfundener Präzedenzfall kann Jahrzehnte fortbestehen, bis er durch „overruling “22 oder durch Intervention des Gesetzgebers („Act of Parliament“) ${ }^{23}$ außer Kraft gesetzt wird. Man kann sich vorstellen, wie sich das Recht an behauptete Änderungen einer Tradition anpassen soll. Es ist kein Zufall, dass die Patentrechte vermeiden, den vergleichsweise engen und präzisen Begriff der „Erfindung " zu definieren ${ }^{24}$ da ist es erst recht praktisch unmöglich, einen exakten, aber gleichzeitig flexiblen Begriff der Tradition oder bestimmter TCEs festzulegen. Wer diese stark eigentumsrechtlich orientierte Argumentation als für den TCE-Schutz ungeeignet kritisiert, muss mit einer funktionierenden Alternative zum Immaterialgüterrechtsschutz aufwarten - denn es sind die soweit bestehenden sui generis-Rechte letztlich doch

20 Hobsbawm 1983, 4, 6-7.

21 Über die generell ahistorische Betrachtung des Common Law als ,ancient and immutable“ im anscheinenden Gegensatz etwa zum afrikanischen Gewohnheitsrecht oder „Customary Law“ siehe auch Chanock 1998, 3.

22 Gillespie/Weare 2017, 94.

23 Dies äußert sich besonders in der sogenannten Mischief Rule der englischen Gesetzesinterpretation (,statutory interpretation“), nachdem bei der Gesetzesauslegung unter anderem berücksichtigt werden soll, dass das Parlament durch Gesetz gegebenenfalls eine unerwünschte Entwicklung des Common Law korrigiert hat, siehe Gillespie/Weare 2017, 42-43.

24 Bainbridge 2012, 432-433 für das englische Recht; Götting 2010, 116 für das deutsche Recht. 
nichts anderes als eigentumsrechtsähnliche exklusive Schutzrechte, sodass die Analogien zum „Intellectual Property“ bis auf Weiteres gültig bleiben.

Ist das TCE-Schutzsystem schon nicht imstande, Traditionen und die ihnen entstammenden TCEs als Schutzobjekt ausreichend zu definieren, so ist es überhaupt völlig ungeeignet, mit Traditionsbrüchen umzugehen. Damit sind wir mitten in der (modernen) Kunst, die schließlich von Traditionsbrüchen lebt. Man kann Werke der modernen Kunst einfach als unter den klassischen Urheberrechtsschutz fallend betrachten, sodass die Frage des TCE-Schutzes gar nicht auftritt, und man sollte diese Interpretation stark befürworten. Denn eine nigerianische Malerin oder Objektkünstlerin oder ein chinesischer Komponist sind Künstler, also Urheber oder Autoren im Sinne des Urheberrechts, ohne Zweifel von ihrer lokalen Kultur beeinflusst (wie ein österreichischer oder irischer Künstler), aber rechtlich gesehen Schöpfer von Werken im Sinne des Urheberrechts und genießen urheberrechtlichen Schutz. ${ }^{25}$ Wie Künstler ihre kulturellen oder indigenen Traditionen benützen, sie konterkarieren, brechen, kritisieren oder ignorieren, ist für das Urheberrecht unwichtig. Aber für einen TCE-Schutz, wenn er tatsächlich wirkungsvoll ist, könnte die Behandlung der Tradition durch moderne Künstler von Bedeutung sein. Kann eine zeitgenössische "indigene“ afrikanische Musikerin auf einem traditionellen Musikinstrument, das traditionell nur Männern vorbehalten ist, traditionelle, oder gar eigene komponierte Musik spielen? Verletzt sie damit nicht die - vielleicht unzweifelhaft bestehende - Tradition ihrer „indigenous community“? Hat ihre „indigenous community“ (früher sagte man „Volksstamm“ oder „tribe“, aber diese rassische Stereotypisierung ist aus der Völkerkunde wenigstens doch endlich verschwunden) einen Rechtsanspruch aus der unangemessenen Nutzung der TCEs ihrer indigenen Kultur, der diesen „Missbrauch" der TCEs in ihrer Kunst unterbindet? Und wer macht den Anspruch geltend? Die (männlichen) Ältesten und Chiefs der Gemeinschaft als anscheinende oder gar anerkannte Repräsentanten der Volksgruppe? ${ }^{26}$

Die ganze, besonders westliche Besorgnis bezüglich des Schutzes von TCEs krankt vor allem an der paternalistischen Haltung, aufgrund der sich Wissenschaftler, Funktionäre internationaler Organisationen und Manager von Unternehmen (denn es geht schließlich auch um das internationale Geschäft mit Ethnoprodukten und Ethnokunst ${ }^{27}$ in ihrer willkürlich

25 ZB Vereinigtes Königreich: Copyright, Designs and Patents Act 1988, $\mathbb{S} 9$ 9(1), 11(1). Frankreich: Code de la Propriété Intellectuelle 1992, Art. L111-1. Deutschland: $\$ \$ \$ 7,11$ UrhG 1965. Österreich: $\mathbb{S} 10(1), 14(1)$ Urheberrechtsgesetz 1936.

$26 \mathrm{Zu}$ dieser Frage siehe unten unter V.

27 Huggan 2001, 13, 35-38, 155-158. 
selbstgestalteten Weise für indigene Völker verantwortlich fühlen wollen, ohne dass sie jemand darum gebeten hat. Dies erscheint als eine Neuauflage des „White Man's Burden“, ${ }^{28}$ allerdings heimtückischerweise oft unter dem Deckmantel einer antikolonialen Haltung. „Ethnische“ Künstler und Künstlerinnen, etwa aus und in Afrika, müssen schon lange nicht mehr ihre "Stimme“ und ihre „Wurzeln" finden, sondern sie schaffen einfach Kunst, die keineswegs ethnisch sein muss (das entscheidet eigenmächtig der Künstler oder die Künstlerin für jedes Werk), sondern ästhetisch oder politisch ist. ${ }^{29}$ So kann ein moderner afrikanischer bildender Künstler sich in seiner Kunst auf seine Tradition beziehen und diese dazu benützen, die Korruption afrikanischer Eliten in der Geschichte, die oft Komplizen des Sklavenhandels waren, ${ }^{30}$ oder die Korruption der Politik in den unabhängigen post-kolonialen Staaten kritisieren. Offensichtlich ist das eine heterodoxe Verwendung seiner Tradition. Wird diese Verwendung als eine Verletzung einer Tradition oder aus ihr erfließenden TCEs gesehen, die einen Unterlassungsanspruch in Hinblick auf seine Kunst ermöglicht, so ist das nichts anderes als Zensur im Widerspruch zur Meinungs- und Kunstfreiheit. ${ }^{31}$ Tatsächlich hat der Künstler den normalen Urheberrechtsschutz für sein Werk, gleichgültig, ob eine politische Aussage darin enthalten ist und diese mit indigenen Traditionen spielt. Es erwachsen dem Künstler auch keine rechtlichen Verpflichtungen gegenüber seiner ethnischen Gruppe in Hinblick auf die Benützung von deren Traditionen und TCEs ${ }^{32}-$ moralische Entrüstung und Indignation hat im Recht keinen Platz, sonst könnte man die ganze (auch westliche) Kunst aufgeben. Der afrikanische Künstler ist insofern nicht anders gestellt als ein „indigener“ polnischer Künstler oder eine aus dem Libanon stammende Künstlerin, die in Paris lebt und sich als französische Künstlerin fühlt. Wenn man den afrikanischen Künstler in ein gezwungenes ethnisches „traditionelles“ Klischee hineinstößt,

28 Kipling 2013, 528. Kiplings Gedicht „The White Man’s Burden“ ist aus dem Jahre 1899. Siehe, von der Seite der Kolonistierten, zB Césaire 2000, 60.

29 Njami 2005a, 21. Siehe auch Mosley 1999, 84-85.

30 ZB der Photograph Samuel Fosso (geb. 1962), Série Tati, Autoportrait II: Le chef: celui qui a vendu l'Afrique aux colons (1997), in: Njami 2005b, 76.

31 Über die Verwendung (vielleicht) westlicher Prinzipien, siehe unten unter VI.

32 Im Gegenteil dazu will die australische Entscheidung John Bulun Bulun and another v. R. \& T. Textiles Pty. Ltd. [1998] FCA 1082 (Federal Court of Australia) eine treuhänderische (fiduciary) Beziehung des indigenen Künstlers zu seinem Volk erkennen, sodass der Künstler indigene Werke in Verletzung indigener Gebräuche und gewohnheitsrechtlicher Regeln nicht verwerten darf. Siehe Helfer/ Austin 2011, 483, 493. 
dem dieser in seiner Kunst zu entsprechen habe, so ist das ganz gewöhnlicher Rassismus.

Die Idee des normierten Andersseins, hier durch anscheinend wohlmeinenden Schutz von TCEs und - regelmäßig nicht-westlichen - Traditionen, mag in einer globalisierten Welt eine Illusion von Identität vermitteln, in die man sich nach Belieben versenken kann, indem man sich zur persönlichen Sinnstiftung für die Rettung indigener Traditionen verantwortlich fühlt, oder, noch eigennütziger, die Erschließung neuer Marktsegmente in Ethnoware vorantreibt. Tatsächlich liegt aber dem TCE- und Traditionsschutz in Kultur und Kunst die hinterhältige Vorstellung einer rechtlich sanktionierten Segregation und Diskriminierung zugrunde, allerdings in einer neuen, schmackhafteren und scheinbar unbedenklichen Form. Angesichts immer stärker werdender identitärer Bewegungen weltweit wird zweifelhaft, ob die Vertreter des TCE-Schutzes immer noch als so naiv angesehen werden können, dass sie in ihrer wohlwollenden Sorge um indigene Völker die Gefahr wirklich nicht erkennen können.

\section{Rechtsschutz von Kunst als (identitärer) Rassismus}

Die eigentumsähnliche Konstruktion der sui generis-TCE-Schutzrechte erfordert Rechteinhaber wie die Eigentümer eines Eigentumsrechts. Gleichzeitig werden Befürworter des TCE-Schutzes nicht müde zu betonen, dass eine individuelle Autorenschaft und damit verbundene Innehabung von ausschließlichen Eigentumsrechten wie in (moderner, jedenfalls nach-mittelalterlicher) westlicher Kultur und Kunst in indigenen Gemeinschaften nicht existiert oder den westlichen Kulturen nicht vergleichbar ist. ${ }^{33}$ Man kann daher die Erzeugnisse indigener traditioneller Kunst schon ihrem Wesen nach nicht spezifischen Schöpfern zuschreiben und daher, wie sonst im Urheberrecht, Rechteinhaber individuell feststellen. Das wird auch mit der Behauptung verknüpft, dass indigene Gesellschaften im Gegensatz zu westlichen Kulturen nicht das individuelle Privateigentum unterstreichen, sondern dass indigenes Vermögen im gemeinschaftlichen Ei-

33 Macmillan 2018, 49-52, Harding 2018, 89-90. In Wahrheit ist die Situation viel komplizierter, und vor allem existiert „indigene“ individuelle Autorenschaft und Kreativität, und wird auch so aufgefasst, siehe zB Diawara 2019, 238, 265 mit Beispielen aus Mali. 
gentum stehe. ${ }^{34}$ Jedenfalls der letzte Punkt ist in dieser Allgemeinheit unrichtig, aber es gibt in dem Bereich sehr wenige verlässliche Forschungen. ${ }^{35}$

Man sollte mit generellen Betrachtungen über nicht-westliche Völker ohnehin vorsichtig sein, damit man nicht in einen Orientalismus (Edward Said) ${ }^{36}$ verfällt (es gibt freilich auch einen korrespondierenden Okzidentalismus), oder sogar einem vulgären Rousseauismus das Wort redet, der nicht-westliche Völker zu edlen gelehrigen Primitiven stilisiert, die dann dankbar die Errungenschaften der höher entwickelten weißen Zivilisation zu empfangen haben. Ein Beispiel ist die Annahme, man dürfe China bei seiner weitverbreiteten Urheberrechts- und Markenpiraterie nicht an den Maßstäben der westlichen Welt messen, weil die chinesische Kultur kein Verständnis für den Schutz eines Werkes gehabt hätte und daher mit der Idee des Plagiats und der Piraterie nichts anfangen könne. ${ }^{37}$ Das ist zwar falsch, ${ }^{38}$ aber dieses falsch konstruierte Bild von chinesischer Kultur wird dann verwendet, um herablassend für das Verständnis nicht-westlicher Kulturen zu werben, diese aber gleichzeitig am westlichen kulturellen Fortschritt des Immaterialgüterschutzes teilhaben zu lassen und damit zu zivilisieren. ${ }^{39}$ Tatsächlich gab es in China immer schon den Begriff des Verbots der unberechtigten Verwendung von Werken..$^{40}$ Was jetzt in China passiert, ist nichts anderes als eine allgegenwärtige Verletzung von Immaterialgüterrechten, und das wird auch in China so verstanden, ohne die Notwendigkeit einer Nachhilfe in westlichen Werten. China verhält sich hier übrigens nicht anders als früher die Vereinigten Staaten, die das gesamte neunzehnte Jahrhundert unbekümmert Raubdrucke besonders von englischer Literatur herstellten, und später ihre eigene Copyright-Industrie

34 ZB Gibson 2005, 44. Tatsächlich ist das Bild besonders heute viel differenzierter, siehe zB Röschenthaler 2019, 201-202, Musangi 2019, 229-240 - diese beiden Texte zeigen viel besser als es hier möglich ist die Komplexitäten und Probleme eines Urheberrechts- und „Folklore“- Schutzes in ,indigenen“ Gemeinschaften in Afrika.

35 Rahmatian 2019, 170-171, mwN.

36 Said 2003, 2-4, 13-15, 22-23, 62-63, 104-106, für Definitionen und Beispiele.

37 ZB Alford 1996-97, 1140-141.

38 Shao 2005, 403, 407, 423-428.

39 In diesem Geist weist zB Absatz 2 der Präambel zum Welthandelsabkommen (Marrakesh Agreement) darauf hin, der Zweck des Abkommens sei, „to ensure that developing countries, and especially the least developed among them, secure a share in the growth in international trade commensurate with the needs of their economic development". Siehe auch die Präambel von TRIPs in Absatz 6.

40 Shao 2005, 414-416. 
mit der „Manufacturing Clause“ schützten - Urheberrechtsschutz in den USA nur dann, wenn das Buch eines ausländischen Autors in den USA gedruckt wurde ${ }^{41}$-, eine Maßnahme, die erst mit dem Beitritt der USA zur Revidierten Berner Übereinkunft 1989 verschwand. ${ }^{42}$ Auch als „brothers in crime" sind westliche und nicht-westliche Kulturen offenbar nicht unähnlich, und die kulturellen Unterschiede scheinen doch nicht so wichtig zu sein.

Da indigene Gemeinschaften (,indigenous communities“) ihr kulturelles Vermögen gemeinschaftlich besitzen sollen, so soll ihnen auch der TCE-Schutz gemeinschaftlich zustehen. ${ }^{43}$ Aber schon der Begriff ,indigenous community" ist teilweise ahistorisch und nimmt nicht Bezug auf Urbanisierung und Wanderarbeiterschaft, die das ganze zwanzigste Jahrhundert existierte. ${ }^{44}$ Und wer genau nimmt die TCE-Rechte im Namen der Gemeinschaft wahr? Die Gemeinschaft ist höchstwahrscheinlich nicht nach westlich-demokratischen Kriterien organisiert, ${ }^{45}$ nach denen jeder und jede, auch das dreizehnjährige Mädchen, vertreten wird, und sein/ihr Vertreter demokratisch legitimiert ist und daher politisch oder rechtlich zur Verantwortung gezogen werden kann. In Wahrheit funktioniert das nicht einmal zuverlässig in westlichen freiheitlichen Demokratien, aber es ist wenigstens ein anzustrebendes Ideal. In nicht-westlichen Gesellschaften existiert dieses Ideal nicht, und es besteht kein Ansporn, delegierte Rechte über die kleine führende Gruppe von Chiefs und Ältesten (oder eine Verwertungsgesellschaft, ${ }^{46}$ oder den Staatspräsidenten $\left.{ }^{47}\right)^{48}$ hinaus an die Allgemeinheit zu verteilen. Immerhin ist dem westlich-liberaler Weltanschauung entstammenden Urheberrecht gleichgültig, ob der Urheber eine drei-

41 Anon 1950, 686.

42 Zur Geschichte des Beitritts der USA zur Revidierten Berner Übereinkunft, siehe Ginsburg/Kernochan 1988, 1-5. Die Regelung der „Manufacturing Clause“ war zum Zeitpunkt des Beitrittes der USA schon ausgelaufen und wurde nicht mehr verlängert.

$43 \mathrm{Vgl}$ oben Kenia, $\$ \$ \$ 9,16$ Protection of Traditional Knowledge and Cultural Expressions Act 2016. Siehe zB. Johnsson 2012, 148-149.

44 Chanock 2009, 180, 188.

45 Über indigene afrikanische politische Systeme, jedoch noch eher aus einem kolonialen Blickwinkel, siehe Fortes/Evans-Pritchard 1940, 14-16, 36-41 [Zulu], 57 [Ngwato], 103-106 [Bemba], 251 [Tallensi].

46 So etwa in der Côte d'Ivoire, Art. 6, Art. 46, Art. 54 Loi n 2016-555 du 26 juillet 2016 relative aux droits d'auteurs et des droits voisins.

47 So etwa in Ghana, siehe $\$ \$ 4(2), 17$ Ghana Copyright Act 2005.

48 Für eine weitere Darstellung der Lösungen in nationalen Einzelrechten in Afrika siehe Nwauche 2017, 54, 67, 98 und, auf dieser Basis, Rahmatian 2019, 165. 
zehnjährige Künstlerin ist: ${ }^{49}$ sie erhält Urheberrechtsschutz; ${ }^{50}$ ob sie ihn in der Realität immer geltend machen kann, steht freilich auf einem anderen Blatt. Einen TCE-Schutz, der einer „indigenous community“ verliehen wird, wird die Dreizehnjährige nicht einmal theoretisch bekommen können. Letztlich ist ein TCE-Schutz ein Freibrief für ausgedehnte Korruption auf lokaler Ebene.

Ist es schon schwierig, die geeigneten Vertreter der indigenen Gemeinschaft als Rechtsträger der TCEs ausfindig zu machen, so kann es noch schwieriger sein, festzustellen, wer eigentlich Mitglied dieser indigenen Gemeinschaft ist und damit potentieller (schon wesentlich seltener aktueller) Nutznießer des TCE-Schutzes sein kann. Die Definition im obigen Beispiel des kenianischen Gesetzes von 2016 ist hier durchaus repräsentativ. Die „traditional community“ sei gekennzeichnet durch gemeinsame Abstammung, das Teilen eines gemeinsamen geographischen Lebensraumes und eine spezifische Art der Lebensgestaltung und durch eine ähnliche Kultur oder Sprache. Diese Definition kann vielleicht bei Gemeinschaften funktionieren, die eher abgeschlossen und ohne viel Kontakt mit der westlichen Zivilisation leben, aber dies wird in der modernen Welt immer seltener. Im Normalfall jedoch führen solche Kriterien letztlich zu einer rassischen Einteilung und Segregation. Es wäre interessant zu erfahren, wie man die Zugehörigkeit zu einer normativ postulierten indigenen Volksgemeinschaft bestimmen soll, vielleicht mit DNA-Tests, um festzustellen, ob man Mitglied dessen sei, was die Nazis noch als "Gemeinschaft des Blutes“ bezeichneten. Überhaupt sind die Vorläufer einer solchen Denkweise, die Rassen(trennungs)gesetzgebungen der Nationalsozialisten und von Apartheid-Südafrika, ${ }^{51}$ von einer vergleichsweise erfrischenden Offenheit; sie sind gewollt rassistisch, ohne dass ein komplizierter Nachweis geführt werden muss, während die Ideen eines TCE-Schutzes in ihrer letzten Konsequenz auch rassistisch sein müssen, dies aber wesentlich schlechter erkennbar ist, weil sie sich für wohlmeinend halten und zum Nutzen der betroffenen Bevölkerung mit anderer Kultur sein wollen. Das scheint auch der

49 Bekanntlich hängt der Urheberrechtsschutz nicht von der Geschäftsfähigkeit des Urhebers ab, siehe etwa Rebbinder 2010, 104-105.

50 Das Charakteristische am Urheberrechtsschutz ist seine Ausrichtung auf Individuen, nicht Kollektive, siehe Schovsbo 2012, 167.

51 Siehe Beispiele in Rabmatian 2019, 172-174, Rabmatian 2007, 225-227. Für eine Darstellung der ideologischen Basis des Apartheidsystems, siehe Dubow 2014, 53-68. 
vorgebliche Ursprung der Idee eines Apartheidssystems gewesen zu sein..$^{52}$ Wohlmeinend scheinend oder nicht, bösartig sind alle solche Konzeptionen und strikt abzulehnen. Im Bereich der Kunst und auch der Wissenschaft ist die Idee eines indigenen, kulturell oder gar rassisch reinen Künstlertums ${ }^{53}$ oder Wissenschaftsbetriebes ohnehin eine gefährliche Wahnvorstellung, ähnlich der Schaffung einer „deutschen Physik“ ohne die Juden Albert Einstein und Max Born, von der die Nationalsozialisten halluzinierten. ${ }^{54}$

Es wäre unrichtig anzunehmen, dass von nicht-westlichen, etwa afrikanischen Ländern erlassene Gesetze nie inhärent rassistisch sein oder zu rassistischen Zwecken missbraucht werden könnten. Im Guten wie im Bösen sind alle Menschen gleich - Rassismus besteht ja unter anderem darin, dass man sich für überlegener, moralisch hochstehender, zivilisierter und wertvoller hält als andere Menschen und Völker, oder auch diese angeblichen Eigenschaften anderen zuschreibt. ${ }^{55}$ Außerdem wurde der Rassismus westlicher Spielart durch die Kolonialreiche ausgiebig in alle Welt exportiert; das koloniale System und die Behandlung der kolonialisierten Völker etwa durch das Britische Kolonialreich war schließlich ein Modell für das Deutsche Kaiserreich, ${ }^{56}$ und beeinflusste auch die Nationalsozialisten, obwohl diese den Imperialismus des italienischen Faschismus als ein für sie relevanteres und positiveres Vorbild betrachteten. ${ }^{57}$

Es fällt auf, dass das Problem des TCE-Schutzes regelmäßig im Zusammenhang mit nicht-westlichen indigenen Völkern (etwa in Afrika) oder nach der Kolonialisierung übriggebliebenen indigenen Gemeinschaften oder „First Nations“ (etwa in Australien, Neuseeland, Nordamerika) auf-

52 Dubow 2014, 10, den späteren südafrikanischen Premierminister Malan aus dem Jahre 1944 zitierend, nachdem Apartheid eine Methode sei „to give the various races the opportunity of uplifting themselves on the basis of what is their own." Vgl. Cell 1982, 28-29. Siehe auch Hellmann 1949, 288, damals schon kritisch über die angebliche Funktion der Pass Laws zum Schutz der Schwarzen in Südafrika in Arbeitsverträgen mit Weißen.

53 Die wirre und bösartige Vorstellung der Nazis von „Geistesjuden“ und „entarteter Kunst" ist hinlänglich bekannt.

54 Fölsing 1995, 599-600, 745.

55 Diskussion von modernen Formen des Rassismus, siehe zB Frederickson 2002, 139-150. Siehe Mosley 1999, 74-78 über die möglicherweise rassistischen Aspekte der „Négritude“ (Mosley verneint dies). Eine frühe kritische Darstellung von rassischen Vorurteilen, Stereotypisierungen und Vorurteilen gegenüber einer Gruppe mit besonderem Bezug auf Südafrika, siehe Hellmann 1949, 683, 697.

56 Lindner 2011, 43-65, Bernhard 2017, 211, 223-224.

57 Bernhard 2017, 208-209. 
ritt, aber kaum jemals in Europa ${ }^{58}$ wo doch auch genügend Völker leben, die eigentlich als ,indigen“ gelten müssten. Man erinnert sich an die Verwendung adaptierter indigener Rechte zur Machtausübung der Kolonialmächte über kolonialisierte indigene Völker, wie etwa durch die Indirect Rule des British Empire. ${ }^{59}$ In Südafrika instrumentalisierte der Natal Native Code von 1932 die umgearbeiteten indigenen Gewohnheitsrechte zum Zwecke weißer Machtausübung über indigene schwarze Gemeinschaften. ${ }^{60}$ Auch TCEs, bei denen die ordnende Macht des Zentralstaates den Vertretern indigener Gemeinschaften Rechte gewährt, um die indigene Kultur und ihre Erzeugnisse zu bewahren, können eine ähnliche Wirkung entfalten. Vielleicht sollen sie dies auch, weil damit politische und soziale Entwicklungen, wie etwa Säkularismus oder die Gleichstellung der Frau, als gegen die kulturelle Tradition gerichtet, verhindert oder verzögert werden können, wobei der Zentralstaat diese Verhinderung politisch geschickt nach außen in indigene Gemeinschaften als Form von „Empowerment" verlagert und damit einer liberal-demokratischen Kritik und Verfassungskontrolle entgeht.

$\mathrm{Ob}$ in der Verfolgung und Ausrottung oder im Schutz, es scheint immer ein weißer westlicher Plan zu sein, der einmal den Unglauben der nicht-weißen Wilden, einmal die christliche Nächstenliebe gegenüber allen Menschen betont, was eben in der Bibel gerade politisch passt: ${ }^{61} \mathrm{im} \mathrm{Er}$ gebnis sind alle solche Aktionen der Ausdruck westlicher kultureller oder rassischer Überlegenheit. Allerdings kommen erhellende Konflikte vor: in Südafrika stand die TCE-Gesetzgebung vor dem Problem, TCE-Schutz eventuell auch weißen Afrikaners und Engländern zugestehen und diese daher als indigene Gesellschaften anerkennen zu müssen oder den TCESchutz nur auf schwarze Gemeinschaften zu beschränken. ${ }^{62}$

Die Frage der Stellung der Weißen in Südafrika bezüglich eines TCESchutzes hat uns nach Europa geführt. Da gibt es die Forderung nach einem Schutz indigener Kultur nämlich auch, nur heißt dies dort anders. Die Neue Rechte, die in vielen europäischen Ländern politisch immer stär-

58 Allerdings nicht immer, siehe das Beispiel Italien und seine Gesetzgebung betreffend einen regionalen Kulturgüterschutz, Petrillo/Scovazzi/Ubertazzi 2019, 193, 197, 201.

59 Chanock 1998, 33, 43-46, 55, 71, 75, 77-78. Besonders für das Verhältnis von Englischem Recht und afrikanischen Gewohnheitsrechten, siehe Allott 1960, 28, 82, 72, 96.

60 Allott 1960, 94-95, Rahmatian 2007, 227 mwN.

61 Vgl. pointiert, aber zutreffend, Césaire 2000, 33

62 Nwauche 2017, 132. 
ker wird, hat die Idee eines Schutzes der eigenen nationalen, autochthonen Kultur und des eigenen Volkes (vielleicht auch der eigenen Rasse) selbstverständlich aufgenommen und knüpft damit nur an extreme rechte Theoretiker des zwanzigsten Jahrhunderts und der Gegenwart an.

Schon Oswald Spengler, der Kulturen als Organismen auffasste, die wachsen und sterben, schloss einen interkulturellen Dialog und einen Einfluss anderer Kulturen aus, weil die Interpretation einer Kultur durch andere Kulturen notwendigerweise oberflächlich bleiben müsse und den der Kultur zugrundeliegenden „Volksgeist" oder „Nationalcharakter" verkenne. ${ }^{63}$ Das zeigt einen Einfluss der Geschichtsphilosophie von Herder, allerdings in einer stark vereinfachenden Interpretation, die für das neunzehnte und zwanzigste Jahrhundert charakteristisch ist. Übrigens gibt es diesen behaupteten Nationalcharakter oft wirklich, aber er entsteht nicht natürlich-mystisch, sondern ist eine willkürliche Erfindung von bestimmten, manchmal sogar identifizierbaren Menschen. ${ }^{64}$ Spengler beeinflusste Alain de Benoist, den wichtigsten Denker der französischen Neuen Rechten und bedeutend für die extreme Rechte in ganz Europa. Benoist attackiert die liberale Idee von individualen Rechten, wie sie die französische Revolution und die amerikanischen Gründerväter verkörperten, und den internationalen Kapitalismus der freien Märkte, weil er die Identitäten der Völker auslösche. Er wendet sich auch gegen den Kosmopolitismus der Linken und Liberalen und unterstützt die Idee von homogenen ethnischen Einheiten, demgemäß jede ethnische Gruppe ihre eigene zu bewahrende Kultur habe, was aber am besten dann gelingt, wenn man unterschiedliche Kulturen in demselben Land vermeidet. ${ }^{65}$ Die Nähe zur Idee des TCE-Schutzes (und der damit verbundenen Rassentrennung) ist offensichtlich. Noch deutlicher werden US-amerikanische Denker, etwa Jared Taylor, der behauptet, rassische Identität ist allen Menschen gemeinsam, daher werden Mitglieder der eigenen Rasse natürlicherweise bevorzugt. Das Gefühl für rassische Unterschiede sei allgemein so stark, dass die Schaffung einer multi-ethnischen Nation die Entwicklung ihrer eigenen Gesellschaft hemme, und auch daher sollten Weiße mehr ethnische Solidarität untereinander zeigen, wie es Nicht-Weiße ja auch tun. ${ }^{6}{ }^{6}$ Weniger intellektuell, aber noch eindeutiger äußert sich etwa Richard B. Spencer, ein wichtiger Vertreter der Identi-

63 Engels 2019, 9, 11.

64 Rahmatian 2016, 918, besonders in Hinblick auf das Problem der Rechtsvereinheitlichung im Urheberrecht in der EU und ein Problem, das ich das „Herdersche Paradox" nenne.

65 Camus 2019, 76-78, 83.

66 Nieli 2019, 139-140, 148. 
tären und der Alt-Right (der Begriff stammt anscheinend von ihm) in den USA und ein "icon for white supremacists“. Sein Ziel ist, „Homelands“ für Weiße zu schaffen, auch um die angebliche kulturelle Enteignung der Weißen durch nicht-weiße Minderheiten und Einwanderer zu verhindern, die von der multikulturellen Gesellschaft und der Rassengleichheit profitieren. Er sieht sich auch als Anhänger der Identiären, wie sie vor allem in Frankreich und Deutschland auftreten, und fordert, wie diese, spezielle Rechte für weiße, europäische ethnokulturelle Gruppen. Europa sei (anscheinend auch für weiße Amerikaner) „our common home, and our ancestors' bone and blood lie in its soil." 67

Und warum ist dieses rechtsextreme Gedankengut strikt abzulehnen, wenn es auf Weiße bezogen ist, aber wünschenswert für Nicht-Weiße „Eingeborene“, denen ein Kulturgüterschutz verabreicht werden soll, in dem doch sehr ähnliches Denken, besonders die inhärente Rassentrennung, zum Ausdruck kommt? Anders ausgedrückt, würde der TCE-Schutz den Unterschied der ethnischen Gruppen als vielleicht gleichartig, aber streng getrennt, betonen, sodass damit die kulturelle Entwicklung jeder Ethnie auf ihre individuelle Art gewährleistet sei, so könnten die meisten Neuen Rechten mit dieser Idee gut leben. Sie ist ja auch nicht neu, es ist die Idee der Apartheid. ${ }^{68}$ Die Forderungen der Neuen Rechten sind schließlich Forderungen für eine Art indigenen Kulturschutz, nur unter einem (teilweise) anderen Namen. Bei genauerer Betrachtung weiß zwar niemand, was nun als Volkszugehörigkeit zum eigenen Volk (oder der eigenen ethnokulturellen Gruppe) zählt und was dieses „Volk“ ist, und wie die autochthone Kultur dieses Volkes aussehen soll, aber das zeigt wieder, dass indigene Kultur und Nation letztlich verordnete, willkürliche normative Begriffe sind, die dann Wirklichkeit werden, wenn die Staatsmacht den politischen Willen hat und stark genug ist, eine solche Kultur aufzuzwingen, wie etwa das Deutschtum im (und durch den) Nationalsozialismus. Ein solcher künstlicher Begriff ist die auch von den Neuen Rechten immer wieder behauptete judeo-christliche Kultur, die dann besonders als Kampfbegriff gegen die is-

67 Bar-On 2019, 225-227, 235.

68 Siehe die Textstellen in Brookes 1968, 1-17 zur Erklärung des Begriffes der Apartheid. Die Arbeitskraft der Schwarzen war allerdings für Weiße gut genug, da konnte die kulturelle Trennung durchbrochen werden. Siehe die Diskussion über die Pass Laws in Südafrika für Schwarze für Arbeitsverträge mit weißen Arbeitgebern (und für die Regulierung des Personenverkehrs) noch vor dem Wahlsieg der Reunited National Party 1948 und der formellen Einführung des Apartheidssystems, Hellmann 1949, 286-292. Siehe auch Cell 1982, 46-51. 
lamische Welt verwendet wird. ${ }^{69}$ Man braucht die Werke der großen klassischen Historiographen des Judentums, wie Heinrich Graetz oder Simon Dubnow ${ }^{70}$ nur durchzublättern, um festzustellen, dass das Verhältnis zwischen Christen und Juden in Europa seit vielen Jahrhunderten wenigstens bis 1945 ein ausschließlich antisemitisches war, und dass die europäische kulturelle Tradition alles getan hat, um niemals eine gemeinsame judeochristliche Kultur entstehen zu lassen. Das westliche „kulturelle Erbe“ ist nicht judeo-christlich, sondern nur christlich und aggressiv gegen die Juden, und in seiner intoleranten Giftigkeit viel näher dem bekämpften militanten Islam als dem angeblich alliierten Judentum.

Auch das Recht ist nicht frei von der Vereinnahmung eines normativen indigenen Kulturbegriffes. Das kann man heute gut etwa am Schottischen Zivilrecht demonstrieren, das als Mischrechtssystem (mixed system - Common Law und Civil Law) gesehen wird, was zwar stimmt, aber, so die herrschende Auffassung unter schottischen Rechtsgelehrten, ein Mischrechtssystem mit speziellen Eigenschaften sei, die eine gewisse Überlegenheit gegenüber anderen Systemen, besonders dem englischen, mit sich bringen sollen. Doch diese Überlegenheit bleibt nicht-schottischen Gelehrten, die außerhalb der schottischen „ethnischen Gruppe“ stehen, seltsamerweise verborgen - selbst solchen wie diesem Autor, der immerhin eine Ausbildung in einem kontinentaleuropäischen und im englischen Recht hat und daher mit den konstituierenden Elementen des schottischen Mischrechts vertraut sein sollte. Dieser „Scottish Legal Nationalism“, wie er sich selbst nennt, versucht eine Art Reinheit des Schottischen Rechts gegen Einflüsse vor allem des englischen Rechts zu bewahren und damit eine kulturelle schottische Integrität, die sich auch im schottischen Recht widerspiegelt, beizubehalten. Auch dieses Konstrukt einer schottischen Rechtstradition kann als eine Variante von TK und TCEs interpretiert werden, deren Nutznießer das schottische Volk als indigene Gemeinschaft sein soll - oder nur die (indigenen schottischen?) Rechtsgelehrten?71

69 Siehe die berechtigte Kritik an der Verwendung dieses Begriffs von Shimon Stein und Moshe Zimmermann, „Weil's besser klingt: Im Begriff ,judeo-christlich“ wird das Jüdische missbraucht, um Forderungen zu legitimieren“, Die Zeit, Nr. 35/2017, 24. August 2017. Antisemitische „Alt Right“ Vertreter, wie etwa Richard B. Spencer, lehnen den Begriff ,judeo-christlich“ als egalitär, multikulturell, liberal und sozialistisch ab, siehe Bar-On 2019, 231.

70 ZB Graetz 1915, Dubnow 1936, 240-273. Sehr instruktiv betreffend das 17. und 18. Jahrhundert ist auch Dubnow 1928. Eine moderne Darstellung zB in Hertz 2018, 737-757, Berkovitz 2018, 924-947, Silber 2018, 788, 791-792.

71 Über den „Scottish Legal Nationalism“ mit direktem Bezug auf TCEs in Rahmatian 2007, 209-210. Eine umfangreiche neuere (und wegen ihrer Kritik in der schot- 
Unter der Überschrift des Schutzes von indigener oder autochthoner Kunst und Kultur kann man ohne Mühen eine identitäre und rassistische Politik und Gesetzgebung aufziehen, die sich vielleicht von der ursprünglichen Zielsetzung, nicht-westliche indigene Kulturen gegen kommerzielle Ausbeutung zu schützen, weit entfernen mag, aber wesensmäßig nicht so fern ist, dass man von einer völligen Pervertierung des ursprünglichen Gedankens sprechen kann. Jede Fragmentierung der Gesellschaft in - anfänglich - vielleicht gesondert zu schützende ethnische oder sonstige Gruppen führt unweigerlich zu einer Unterscheidung, die irgendwann immer in eine Diskriminierung dieser Gruppen durch die politisch und wirtschaftlich mächtigere Mehrheit münden wird, weil sich der Schutzzaun zu einem Käfig für die Geschützten verwandeln wird. Derartige Einteilungen unterminieren vor allem das aufklärerische und liberale Prinzip der Gleichheit vor dem Gesetz.

\section{Ausblick: Ein westliches Verbot westlicher Herrschaftsmittel?}

Der Schutz von TCEs („Traditional Cultural Expressions“) mag einer guten Intention entstammen, aber seine rechtliche Durchsetzung führt notwendigerweise zu einem künstlichen, normativ festgelegten Begriff der Tradition, weil die Anerkennung von spezifischen TCE immer auch eine statische Legaldefinition dessen, was rechtlich als TCE anerkannt wird, mit sich bringt. Diese Anerkennung und der damit verbundene Schutz bedeuten Unterlassungs- und Schadensersatzansprüche als Ausdruck von Exklusivrechten, die den klassischen Eigentumsrechten nachgebildet sind, und damit Einschränkungen besonders für moderne Kunst bedeuten können, die, oft auch willkürlich und als Folge kultureller und rassischer Stereotypisierung, als „ethnisch“ oder „traditionell“ eingestuft wird. Des weiteren ist eher unklar, wer der Anspruchsberechtigte eines TCEs-Schutzes ist, denn die „indigenous community“ ist ein verschwommener Begriff, und praktisch muss der Rechtsschutz von Repräsentanten wahrgenommen werden, die durchaus nicht nach freiheitlich-demokratischen Prinzipien in diese Position gelangt sind, wodurch der mildtätige TCE-Schutz eigentlich unerwünschte autoritäre Strukturen festigt. Außerdem muss man die $\mathrm{Zu}-$

tischen Lehre anscheinend wenig beliebte) Diskussion des schottischen „Mixed System“, wie er durch den "Scottish Legal Nationalism" interpretiert wird, von Rabmatian 2017, 843 mit Quellen bezüglich der Vertreter dieses „Scottish Legal Nationalism.“ 
gehörigkeit zur geschützten „indigenous community“ genau genug bestimmen, und diese Aufgabe gleitet schnell zu berüchtigten rassischen und segregierenden Kriterien herab, die aus der Geschichte des neunzehnten und zwanzigsten Jahrhunderts nur zu gut bekannt sind.

Ein Rechtsinstitut, das weder den Schutzgegenstand noch die Berechtigten und Nutznießer des Schutzes zureichend genau bestimmen kann, wäre daher schon nach rechtsstaatlichen Gesichtspunkten suspekt. Was hier freilich präsentiert wurde, ist eine ungeschminkte Darstellung aller Konsequenzen eines TCE-Schutzes, der auch funktioniert. Das ist aber - glücklicherweise - nicht der Fall, und nach dem zuvor Gesagten muss man hoffen, dass Gesetzgebungen im Bereich der TCEs weiterhin möglichst wenig Wirkung entfalten. Es scheint ein politisches Bedürfnis für Ankündigungsgesetzgebungen zu geben, aber wenn diese im Wesentlichen unwirksam bleiben, so ist dies im Fall des TCE-Schutzes besonders für die Förderung und den Schutz moderner Kunst zu begrüßen.

Man kann einwenden, dass die gegenwärtige Argumentation wieder nur eine Neuauflage eines weißen, westlichen paternalistischen Anspruchs ist, die nicht-westliche Welt zu belehren, was sie zu tun habe: anstatt eines anscheinend nicht-westlichen TCE-Schutzes doch kein TCE-Schutz, um westliche freiheitlich-demokratische Prinzipien, wie etwa die verfassungsrechtlich gewährleistete Meinungs- und Kunstfreiheit, und die Gleichheit aller vor dem Gesetz, nicht zu gefährden. Dieser Einwand ist begründet, aber man muss sich fragen, ob etwa Grund- und Freiheitsrechte, der Schutz des menschlichen Lebens und der Würde der Person, wirklich nur auf Errungenschaften der westlichen Zivilisation zurückzuführen sind. Zur Zeit der Allgemeinen Erklärung der Menschenrechte der UNO 1948 scheint man die Universalität bestimmter Grundrechte angenommen zu haben (die Präambel spricht von „Anerkennung der angeborenen Würde und der gleichen und unveräußerlichen Rechte aller Mitglieder der Gemeinschaft der Menschen ... ein gemeinsames Verständnis dieser Rechte und Freiheiten"), während man heute, überspitzt gesagt, mit dem Argument durchkommen soll: „Bei uns hat sich die Frau dem Mann unterzuordnen - respektiert unsere Kultur!“

Man sollte klassische liberale, universalistische Rechtsschutzmechanismen, denen der gewöhnliche Urheberrechtsschutz angehört, nicht zu schnell verteufeln; sie sind oft das einzige, was einer Minderheit bleibt. Ein extremes Beispiel aus der jüngeren Geschichte kann dieses Argument illustrieren. Im Frühjahr 1938, als Österreich durch den „Anschluss“ Bestandteil des nationalsozialistischen Deutschland wurde, kündigten viele Vermieter in Wien ihre Mieter deshalb, weil diese Juden waren. Einige dieser Mieter gingen vor Gericht, und während viele Urteile den Vermietern 
Recht gaben, erklärten andere Entscheidungen diese Kündigungen als unwirksam im Sinne des Mietengesetzes 1922, da die prinzipielle Ablehnung einer Mietpartei allein (besonders dass ein Mieter Jude war), kein anerkannter Grund im Sinne des (liberalen) Gesetzes für eine Kündigung des Vermieters war. ${ }^{72} \mathrm{Um}$ sicherzustellen, dass der liberale Rechtsschutz endgültig unwirksam werde, reagierte die nationalsozialistische Gesetzgebung daraufhin im Mai 1939 mit einer Verordnung, die jeglichen Schutz von Juden vor Räumungen beseitigte, ${ }^{73}$ was man gemäß der Idee des TCE-Schutzes als spezifischen Ausdruck deutscher Kultur und daher als nicht zu beanstanden deuten könnte.

Es ist jedenfalls unrichtig, anzunehmen, dass der westliche Kolonialismus den kolonisierten Völkern die westliche Kultur gebracht habe, etwa, um das Wohlergehen der kolonisierten Bevölkerung zu verbessern $;{ }^{74}$ eine solche Verbesserung, wenn überhaupt, kommt nur insofern in Frage, wenn es die (besonders wirtschaftlichen) Interessen der Kolonialherren fördert. ${ }^{75}$ Eine Einführung von westlichen Grund- und Freiheitsrechten, einschließlich der Kunstfreiheit (wenn diese überhaupt vorwiegend westliche Werte sein sollen) fand daher ohnehin nicht statt, und eine Ablehnung dieser Prinzipien, um eine (Neo-)Kolonialisierung zu vermeiden, wäre schon aus diesem Grund verfehlt. Es kommt auch darauf an, was die Kolonialherren eingeführt haben: die Gesetze der Rassentrennung waren auch „westlich“, galten aber viel prononcierter in den Kolonien als in den Mutterländern England und Frankreich als den Quellen jener westlichen Kulturen. In den Vereinigten Staaten kamen diese beiden Welten zusammen, und dies veranlasste James Baldwin im Jahre 1963 zu unterstreichen, dass, anders als wohlgesonnene Sozialreformer es vielleicht wünschten, es nicht darum gehe, Schwarze auf das Niveau der (weißen) amerikanischen Zivilisation hinzuführen, sondern: ${ }^{76}$

"The only thing white people have that black people need, or should want, is power - and no one holds power forever ... In short, we, the black and the white, deeply need each other here if we are really to become a nation ... There is absolutely no reason to suppose that white people are better equipped to frame the laws by which I am to be gov-

72 Graf 2004, 28.

73 GBlÖ 1939/607. Graf 2004, 25.

74 Vgl. Césaire 2000, 33-36.

75 Baldwin, „Nobody knows my Name: Princes and Powers“, 1998, 155-156, Aimé Césaire zitierend.

76 Baldwin, „The Fire next Time: Down at the Cross“, 1998, 341-342. 
erned than I am. It is entirely unacceptable that I should have no voice in the political affairs of my own country, for I am not a ward of America; I am one of the first Americans to arrive on these shores."

Nirgendwo lehnt Baldwin die Prinzipien der amerikanischen Verfassung $a b$, sondern er verlangt dieselben verfassungsmäßigen Rechte wie die Weißen, aber nicht verliehen von jenen Weißen aus Wohltat und Güte, sondern aus eigenem natürlichem Recht. Die verfassungsmäßigen Rechte sind daher nicht „weiß“, sondern bleiben generell gültig, ohne zwingende Assimilation und Aufgabe der eigenen Kultur. Das dürfte im amerikanischen Civil Rights Movement allgemein so verstanden worden sein, wie eine gemäßigte und eine radikale Quelle zeigt. Martin Luther King sagte: ${ }^{77}$

„I would like to ask those people who seek to apportion to us the rights they have always enjoyed whether they believe that the framers of the Declaration of Independence intended that liberty should be divided into instalments, doled out on a deferred-payment plan. Did not nature create birth as a single process? Is not freedom the negation of servitude?"

Und bei Malcolm X finden wir: ${ }^{78}$

„America is worse than South Africa, because not only is America racist, but she also is deceitful and hypocritical. South Africa preaches segregation and practices segregation.... America preaches integration and practices segregation. She preaches one thing while deceitfully practicing another."

Die Prinzipien, auf die sich die Autoren hier berufen, sind vielleicht „westlich“, aber beklagt wird nicht dieses, sondern die Doppelmoral, was sinnlos wäre, wenn ein sogenannter „separate but (perhaps) equal“-Grundsatz gelten sollte, den aber unter anderem ein TCE-Schutz verordnen würde.

Soll daher überhaupt kein Schutz traditioneller Kulturen und Erzeugnisse bestehen? Man braucht einen solchen Schutz nicht auszuschließen, aber er wird am ehesten ein verfassungsrechtlich verbriefter Individualschutz, nicht ein nebuloser Kollektivschutz sein können. Wenn die Würde des Menschen geschützt werden soll, so auch seine Überzeugungen, Kultur, Tradition, Religion oder Abwesenheit von Religion - aber nicht als Selbstzweck, sondern als Ausdruck des Schutzes der Menschenwürde des/der individuellen Beschwerdeführer/in. Wenn es einen Konflikt etwa

77 King, Why We Can't Wait, 1964, 128.

78 Malcolm X, An Appeal to African Heads of State, 1990, 75. 
mit der Meinungsfreiheit oder der Kunstfreiheit gibt, so wird eine Güterabwägung notwendig werden, mit der die Verfassungsgerichtsbarkeit bestens vertraut ist.

Zum Abschluss sei daran erinnert, dass man das Problem nicht mit einem oberflächlichen Wertrelativismus aus der Welt schaffen kann. Es gibt doch einige zentrale universalistische Grundideen. Oder, wie es Paul Feyerabend formuliert: ${ }^{79}$

„Wenn ich mir ansehe, wieviel die Kulturen voneinander gelernt und wie unbefangen sie das gesammelte Material übernommen haben, dann komme ich zu dem Schluss, dass jede Kultur potentiell alle Kulturen in sich birgt und dass bestimmte kulturelle Züge nichts anderes sind als die wandelbaren Ausdrucksformen einer einzigen menschlichen $\mathrm{Na}$ tur."

\section{Literaturverzeichnis}

Alford, William P. (1996-97), „Making the World Safe for what? Intellectual Property Rights, Human Rights, and Foreign Economic Policy in the Post-European Cold War World“, 29 New York University Journal of International Law and Policy, pp. 135-152

Allott, Antony (1960), Essays in African Law, with special reference to the law of Gha$n a$, London: Butterworth \& Co.

Anon. (1950), „The Manufacturing clause: Copyright protection to the foreign author", 50(5) Columbia Law Review, pp. 686-699

Bainbridge, David I. (2012), Intellectual Property, 9. Aufl., Harlow, Essex: Pearson Education

Baldwin, James (1998), Collected Essays, Hrsg. Toni Morrison, New York: The Library of America

Bar-On, Tamir (2019), „Richard B. Spencer and the Alt Right“, in: Mark Sedgwick (Hrsg.), Key Thinkers of the Radical Right: Behind the New Threat to Liberal Democracy, Oxford: Oxford University Press

Berkovitz, Jay R. (2018), „The Jews of France (1650-1815)“, in: Jonathan Karp and Adam Sutcliffe (Hrsg.), The Cambridge History of Judaism, Vol. 7: The early modern World, 1500-1815, Cambridge: Cambridge University Press, pp. 923-948

Bernhard, Patrick (2017), „Colonial crossovers: Nazi Germany and its entanglements with other empires", 12 Journal of Global History, pp. 206-227

Brookes, Edgar H. (1968), Apartheid: A Documentary Study of Modern South Africa, London: Routledge \& Kegal Paul

79 Feyerabend 2016, 205 (im Original kursiv). 
Camus, Jean-Yves (2019), „Alain de Benoist and the New Right“, in: Mark Sedgwick (Hrsg.), Key Thinkers of the Radical Right: Behind the New Threat to Liberal Democracy, Oxford: Oxford University Press

Cell, John W. (1982), The Highest Stage of White Supremacy: The Origins of Segregation in South Africa and the American South, Cambridge, New York: Cambridge University Press

Césaire, Aimé (2000), Discourse on Colonialism, New York: Monthly Review Press

Chanock, Martin (1998), Law, Custom and Social Order: The Colonial Experience in Malawi and Zambia, Portsmouth, NH: Heinemann

Chanock, Martin (2009), „Branding Identity and Copyrighting Culture: Orientations towards the Customary in Traditional Knowledge Dis-course," in: Christoph Antons (Hrsg.), Traditional Knowledge, Traditional Cultural Expressions and Intellectual Property Law in the Asia-Pacific Region, Alphen aan den Rijn: WoltersKluwer, pp. 177-193

Diawara, Mamadou (2019), „Breaking the contract? Handling intangible cultural goods among different generations in Mali“, in: Ute Röschenthaler und Mamadou Diawara (Hrsg.), Copyright Africa: How intellectual property, media and markets transform immaterial cultural goods, Canon Pyon, Herefordshire: Sean Kingston Publishing, pp. 246-269

Drahos, Peter und Frankel, Susy (2012), „Indigenous People's Innovation and Intellectual Property: The Issues, “ in: Peter Drahos und Susy Frankel (Hrsg.) Indigenous People's Innovation, Canberra: ANU Press, pp. 1-28

Dubnow, Simon (1928), Weltgeschichte des jüdischen Volkes, Bd. 7: Neuzeit. Zweite Hälfte des XVII. und das XVIII. Jahrbundert, Berlin: Jüdischer Verlag

Dubnow, Simon (1936), A Short History of the Jewish People, London: M. L. Cailingold

Dubow, Saul (2014), Apartheid, 1948-1994, Oxford: Oxford University Press

Dutfield, Graham (2017), 'TK unlimited: the emergence but incoherent international law of traditional knowledge protection', 20 Journal of World Intellectual Property, 144-159

Engels, David (2019), „Oswald Spengler and the Decline of the West“, in: Mark Sedgwick (Hrsg.), Key Thinkers of the Radical Right: Behind the New Threat to Liberal Democracy, Oxford: Oxford University Press

Feyerabend, Paul (2016), Zeitverschwendung, Frankfurt am Main: Suhrkamp

Fölsing, Albrecht (1995), Albert Einstein: Eine Biographie, Frankfurt am Main: Suhrkamp Taschenbuch Verlag

Forsyth, Miranda (2012), „Do You Want it Gift Wrapped? Protecting Traditional Knowledge in the Pacific Island Countries“, in: Peter Drahos und Susy Frankel (Hrsg.) Indigenous People's Innovation, Canberra: ANU Press, pp. 189-214

Fortes, Meyer und Evans-Pritchard, Edward E. (Hrsg.) (1940), African Political Systems, Oxford: Oxford University Press

Frederickson, George M. (2002), Racism: A Short History, Princeton, NJ: Princeton University Press 
Gibson, Johanna (2005), Community Resources. Intellectual Property, International Trade and Protection of Traditional Knowledge, Aldershot: Ashgate

Gillespie, Alisdair, A. und Weare, Siobhan (2017), The English Legal System, 6. Aufl., Oxford: Oxford University Press

Ginsburg Jane C. and Kernochan, John M. (1988), „One Hundred and Two Years Later: The U.S. Joins the Berne Convention“, 13 No. 1 Columbia-VLA Journal of Law \& the Arts, pp. 1-38

Girsberger, Martin und Müller, Benny (2012), 'International trade in indigenous cultural heritage: an IP practitioners' perspective', in: Christoph B. Graber, Karolina Kuprecht und Jessica C. Lai (Hrsg.), International Trade in Indigenous Cultural Heritage: Legal and Policy Issues, Cheltenham: Edward Elgar, pp. 175-195

Götting, Horst-Peter (2010), Gewerblicher Rechtsschutz, 9. Aufl., München: C. H. Beck

Graetz, Heinrich (1915), Volkstümliche Geschichte der Juden, Band 3, Wien, Berlin: R. Löwit

Graf, Georg (2004), „Der Entzug von Mietrechten“, in: Georg Graf, Brigitte BailerGalanda, et al., „Arisierung“ und Rückstellung von Wohnungen in Wien, Wien, München: Oldenbourg Verlag, pp. 11-89 (Veröffentlichungen der Österreichischen Historikerkommission. Vermögensentzug während der NS-Zeit sowie Rückstellungen und Entschädigungen seit 1945 in Österreich, Vol. 14)

Hallett, Robin (1974), Africa since 1875: A Modern History, Ann Arbor: The University of Michigan Press

Harding, Sarah (2018), „Contemporary ICH (Intangible Cultural Heritage) and the right to exclude, “ in: Charlotte Waelde et al. (Hrsg.), Research Handbook on Contemporary Intangible Cultural Heritage: Law and Heritage, Cheltenham, Edward Elgar, pp. 78-104

Helfer, Laurence R. und Austin, Graeme W. (2011), Human Rights and Intellectual Property. Mapping the Global Interface, Cambridge: Cambridge University Press

Hellmann, Ellen (Hrsg.) (1949), Handbook on Race Relations in South Africa, Cape Town; London: Oxford University Press

Hertz, Deborah (2018), „Judaism in Germany (1650-1815)“, in: Jonathan Karp and Adam Sutcliffe (Hrsg.), The Cambridge History of Judaism, Vol. 7: The early modern World, 1500-1815, Cambridge: Cambridge University Press, pp. 737-762

Hobsbawm, Eric (1983), „Introduction: Inventing Tradition“, in: Eric Hobsbawm und Terence Ranger (Hrsg.), The Invention of Tradition, Cambridge: Cambridge University Press, pp. 1-14

Huggan, Graham (2001), The Postcolonial Exotic: Marketing the Margins, London: Routledge

King, Martin Luther, Jr. (1964), Why We Can't Wait, New York: Signet Books, The New American Library

Kipling, Rudyard (2013), The Cambridge Edition of the Poems of Rudyard Kipling, Band 1, Thomas Pinney (Hrsg.), Cambridge: Cambridge University Press

Lindner, Ulrike (2011), Koloniale Begegnungen: Deutschland und Großbritannien als Kolonialmächte in Afrika, 1880-1914, Frankfurt, New York: Campus Verlag 
Macmillan, Fiona (2018), „Contemporary ICH (Intangible Cultural Heritage): between community and market," in: Charlotte Waelde et al. (Hrsg.), Research Handbook on Contemporary Intangible Cultural Heritage: Law and Heritage, Cheltenham, Edward Elgar, pp. 35-53

Malcolm X (1990), Malcolm X Speaks, Hrsg. George Breitman, New York: Grove Press

Mosley, Albert G. (1999), „Negritude, Nationalism and Nativism: Racists or Racialists?", in: Leonard Harris (Hrsg.), Racism, Amherst, New York: Humanity Books, pp. 74-86

Musangi, Neo (2019), „From communal practice to intellectual property: The $\mathrm{Ng}$ qoko Cultural Group, political claim-making and the judicialisation of performance in South Africa“, in: Ute Röschenthaler und Mamadou Diawara (Hrsg.), Copyright Africa: How intellectual property, media and markets transform immaterial cultural goods, Canon Pyon, Herefordshire: Sean Kingston Publishing, pp. 218-245

Nieli, Russell (2019), „Jared Taylor and White Identity“, in: Mark Sedgwick (Hrsg.), Key Thinkers of the Radical Right: Behind the New Threat to Liberal Democracy, Oxford: Oxford University Press

Njami, Simon (2005a), 'Chaos and Metamorphosis', in: Simon Njami (Hrsg.) Africa Remix: Contemporary Art of a Continent, London: Hayward Gallery, pp. 13-23

Njami, Simon (Hrsg.) (2005b), Africa Remix: Contemporary Art of a Continent, London: Hayward Gallery

Nwauche, Enyinna (2017), The Protection of Traditional Cultural Expressions in Africa, Cham: Switzerland, Springer International Publishing AG

Petrillo, Luigi, Scovazzi, Tullio, Ubertazzi, Benedetta (2019), „The Legal Protection of Intangible Cultural Heritage in Italy“, in: Pier Luigi Petrillo (Hrsg.), The Legal Protection of the Intangible Cultural Heritage, Cham: Springer International Publishing, pp. 187-227

Petrillo, Pier Luigi (2019), „Intangible Cultural Heritage and Comparative Law. Towards a Global Legal Protection of the Intangible Cultural Heritage“, in: Pier Luigi Petrillo (Hrsg.), The Legal Protection of the Intangible Cultural Heritage, Cham: Springer International Publishing, pp. 231-263

Rahmatian, Andreas (2007), „Universalist Norms for a Globalised Diversity: on the Protection of Traditional Cultural Expressions“, in: F. Macmillan (Hrsg.) New Directions in Copyright Law, vol. 6 (Cheltenham: Edward Elgar Publishing, 2007), pp. 199-231

Rahmatian, Andreas (2016), „European copyright inside or outside the European Union: pluralism of copyright laws and the ,Herderian paradox ““, 47(8), International Review of Intellectual Property and Competition Law, 912-940

Rahmatian, Andreas (2017), „The political purpose of the ,mixed legal system“ conception in the law of Scotland“", 24(6) Maastricht Journal of European and Comparative Law, 843-863

Rahmatian, Andreas (2019), „Das konzeptionelle Problem des Rechtsschutzes für traditionelles Wissen und traditionelle Kultur“, 52(2) Kritische Justiz, 159-175 
Rehbinder, Manfred (2010), Urbeberrecht, 16. Auflage, München: C. H. Beck

Röschenthaler, Ute (2019), „Be faster than the pirates: copyright and the revivival of ,traditional dances" in south-west Cameroon", in: Ute Röschenthaler und Mamadou Diawara (Hrsg.), Copyright Africa: How intellectual property, media and markets transform immaterial cultural goods, Canon Pyon, Herefordshire: Sean Kingston Publishing, pp. 181-217

Said, Edward W. (2003), Orientalism, London: Penguin

Schovsbo, Jens (2012), „The necessity to collectivize copyright - and dangers thereof", in: Jan Rosén (Hrsg.), Individualism and Collectiveness in Intellectual Property Law, Cheltenham, UK: Edward Elgar, pp. 166-191

Shao, Ken (2005), „An alien of copyright? A reconsideration of the Chinese historical episodes of copyright“, Intellectual Property Quarterly, 4, pp. 400-431

Silber, Michael K. (2018), „The Making of Habsburg Jewry in the long Eighteenth Century“, in: Jonathan Karp and Adam Sutcliffe (Hrsg.), The Cambridge History of Judaism, Vol. 7: The early modern World, 1500-1815, Cambridge: Cambridge University Press, pp. 763-797

Traore, Sidi (2019), „Safeguarding the Intangible Cultural Heritage in Burkina Faso", in: Pier Luigi Petrillo (Hrsg.), The Legal Protection of the Intangible Cultural Heritage, Cham: Springer International Publishing, pp. 117-133

Zografos Johnsson, Daphne (2012), „The Branding of Traditional Cultural Expressions: To Whose Benefit?"“, in: Peter Drahos und Susy Frankel (Hrsg.) Indigenous People's Innovation, Canberra: ANU Press, pp. 147-163 